\title{
Film thickness measurement for elongated bubble flow in microchannel using LIF
}

\author{
German V. Bartkus ${ }^{1,2 a}$ and Vladimir V. Kuznetsov ${ }^{1,2}$ \\ ${ }^{1}$ Kutateladze Institute of Thermophysics SB RAS, Novosibirsk, Russia \\ ${ }^{2}$ Novosibirsk State University, Novosibirsk, Russia
}

\begin{abstract}
Two-phase gas-liquid flow in microchannels occurs in a variety of modern industrial applications. Using high-speed video recording, laser scanning and method LIF the characteristics of upward gas-liquid flow in rectangular microchannels with inlet T-mixer are considered. Statistical characteristics of gas-liquid flow and distributions of the phases in crosssection of a microchannel, with hydraulic diameter less than the capillary constant, were identified in a wide range of liquid and gas flow rates. Experiments were carried out for the flow of water-nitrogen in microchannels with cross section 420x280 $\mu \mathrm{m}$ and 720x1500 $\mu \mathrm{m}$.
\end{abstract}

\section{Introduction}

Multiphase microsystems based on microchannels are of great interest for a variety of applications. Typical dimensions of the channels in these microsystems are in the range from hundreds of nanometers to hundreds of micrometers, which ensures the excellent heat and mass transfer properties and continuous operation mode. Studies of gas-liquid flows in microchannels have shown that it is possible to identify the basic flow patterns: isolated bubbles, Taylor bubbles, slug-annular, annular and churn flow which properties have been well studied $[2,3]$. Important parameters for optimizing the heat and mass transfer processes in microchannels are the length of the bubble, the liquid film thickness and their distribution. Laser scanning was used in [4, 5] for determination of the statistical characteristics of the gas-liquid flows. The liquid film thickness for the Taylor bubbles in square and rectangular capillaries was measured in $[6,7]$. It was shown that the dimensionless film thickness in round and rectangular microchannel has different dependence on the capillary number.

The main objective of the present work is experimental study of the multi-scale upward gas-liquid flow characteristics in rectangular microchannel of the microfluidic devices. The statistical parameters of the flow were defined and local film thickness was measured in microchannels with cross section 420x280 $\mu \mathrm{m}$ and 720x1500 $\mu \mathrm{m}$ using dual laser scanning and laser-induced fluorescence method. Distilled water and nitrogen were used as the working fluids.

\section{Experimental facility and procedure}

The experimental setup for the study of the upward two phase gas-liquid flow in a rectangular microchannel is shown in Figure 1 (a). The nitrogen is used as gas phase which is fed from a tank (1)

\footnotetext{
${ }^{\text {a } C o r r e s p o n d i n g ~ a u t h o r ~: ~ g e r m a n b a r t k u s @ g m a i l . c o m ~}$
} 


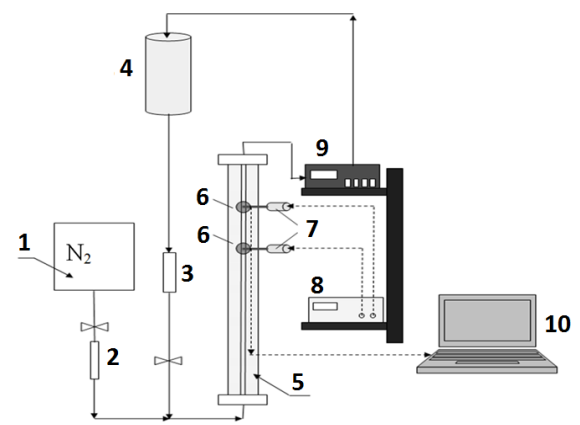

(a)

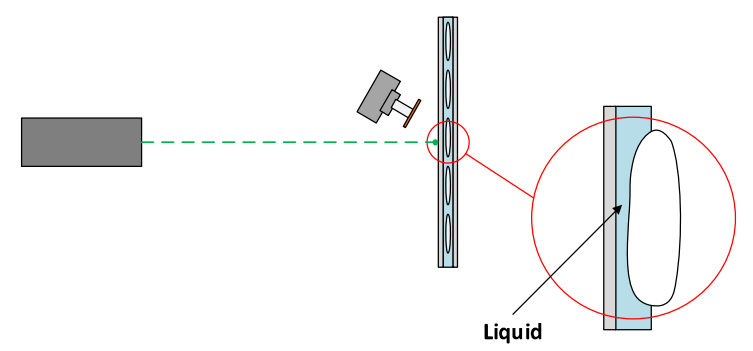

(b)

Figure 1. Experimental setup and scheme of the LIF method application.

via the control valve and gas flow meter (2) to the test section (5). Water flows from the tank (4) through flow meter to the control valve (3) and further into T-shaped mixer at the inlet of the experimental section. Experimental section is a microchannel, consisted from glued glass plates of length $\mathrm{L}=50 \mathrm{~cm}$ with gap, which size is much smaller than the capillary constant $(420 \times 280 \mu \mathrm{m}$, $720 \times 1500 \mu \mathrm{m})$. From the exit of experimental section, the gas-liquid mixture is moved by a peristaltic pump (9) to the water tank (4) where the gas goes to the atmosphere, and saturated water is returned to the experimental section. All experiments were carried out at temperatures between 19 and $21^{\circ} \mathrm{C}$.

The study of the gas-liquid flow patterns was conducted using the method of dual laser scanning, see Figure 1 (a). Two lasers (7) are positioned for illumination of one side of the rectangular channel and the apertures are used to reduce the diameter of the laser beams. The lasers are powered using voltage source (8). The intensity of transmitted light is measured by the photodiodes (6) installed at the opposite side of the channel. Electric voltage from the photodiode is analyzed using high-speed $\mathrm{ADC}$ and processed on the computer (10) over time frame from one to three minutes. Flow patterns are registered using digital video camera. Using laser-induced fluorescence (LIF) method (see Fig. 1 (b)), which provides high spatial resolution without making hydrodynamic disturbances, the distribution of local thickness of liquid film along the long side of the channel was measured for elongated bubbles flow and transition flow. Rhodamine 6G was used as a fluorescent dye.

\section{Flow patterns for upward gas-liquid flow}

Gas-liquid flow in microchannels is accompanied by wide variety of phenomena that are not typical for the large scale. High speed video shows that the periodic flow with elongated gas bubbles (Taylor bubbles), the transition flow and annular flow are the main patterns of the flow for microchannels with the gap substantially less than the capillary constant. In the microchannel with cross section $720 \times 1500$ $\mu \mathrm{m}$, the flow with elongated bubbles and stable liquid plugs is characterized by periodicity and observed in the range of liquid superficial velocities $\mathrm{J}_{\text {liq }}=0.07 \div 0.407 \mathrm{~m} / \mathrm{s}$ and gas superficial velocities $\mathbf{J}_{\text {gas }}=0.04 \div 1.0 \mathrm{~m} / \mathrm{s}$. Superficial velocity was calculated as if the liquid or gas were the only one flowing in a channel cross sectional area. Increasing of the superficial gas velocity above the specified values leads to destruction of the elongated bubbles and the flow loses its periodicity. Figure 2 shows the photograph of the flow at the beginning of elongated bubbles destruction (b) and the corresponding signal from the photodiode (a), in which the existence of the gas phase corresponds to the lowest electric voltage. Such flow is the transition between periodic flow with elongated bubbles and annular flow.

Statistical analysis of the duration of gas and liquid phase existence at photodiode, conducted in the assumption of lognormal distribution of measured values, allows us to propose new method for determination of the flow pattern. Figure 2 (c) shows the square root from overall variance for the duration of gas and liquid phase existence at second photodiode depending on superficial gas velocity. The liquid superficial velocity equals to $0.17 \mathrm{~m} / \mathrm{s}$. The data shows that the periodic flow with elongated bubbles correspond to low values of the variance. After reaching the threshold value of the 


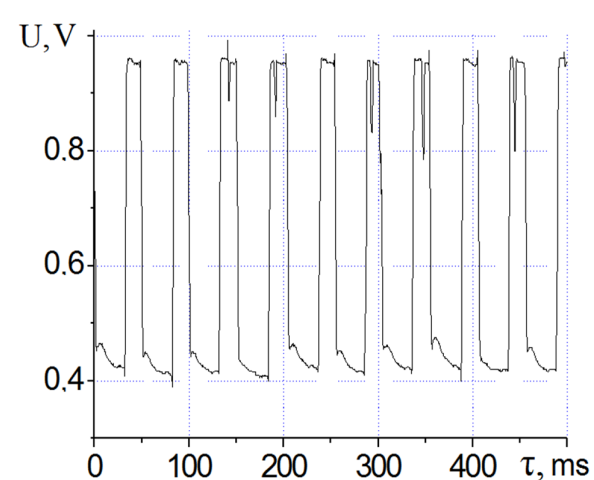

(a)

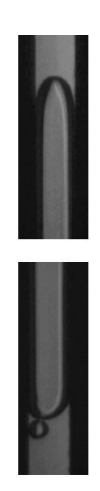

(b)

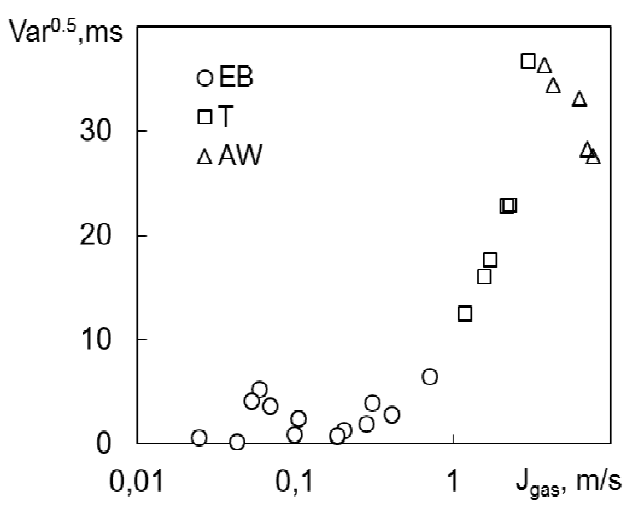

(c)

Figure 2. Dynamics of voltage on the photodiode - (a) and corresponding photo of the flow $-(\mathrm{b})$ for $J_{\text {liq }}=$ $0.27 \mathrm{~m} / \mathrm{s}, J_{\text {gas }}=0.61 \mathrm{~m} / \mathrm{s} ;$ overall variance of the signal from second photodiode for $J_{\text {liq }}=0.17 \mathrm{~m} / \mathrm{s}-(\mathrm{c})$.

superficial gas velocity equals to $1.55 \mathrm{~m} / \mathrm{s}$, the overall variance increases significantly. It shows change in the flow pattern and transformation to transition flow. The highest values of the overall variance correspond to the superficial gas velocities at which complete overlap of the channel cross section by liquid plugs and partial overlap of the channel cross section by disturbance waves on the liquid film surface exist simultaneously. Therefore, the transformation to annular flow corresponds to the maximum in the overall variance because in this flow it decreases due to disappearing of the liquid plugs and reducing the intensity of disturbance waves.

\section{Local liquid film thickness measurements}

The amount of liquid which left on channel wall behind the elongated bubble head gives the important information for analysis of the heat and mass transfer in microchannels. It is known that for capillary tube the film thickness $\delta$ is a monotonic increasing function of the capillary number $C a=U_{b} \mu / \gamma$ and experimental data were fitted in [8] to obtain the empirical equation named the Taylor law:

$$
\delta / D_{h}=0.67 C a^{2 / 3} /\left(1+3.35 C a^{2 / 3}\right) .
$$

Here $D_{\mathrm{h}}$ is the hydraulic diameter of the channel, $U_{\mathrm{b}}$ is the bubble velocity, $\mu$ is the dynamic liquid viscosity and $\gamma$ is the surface tension. The liquid film in a rectangular microchannel consists of a thin liquid layer at the channel wall and liquid filled the channel corners. Using laser-induced fluorescence (LIF) method, which provides high spatial resolution without making hydrodynamic disturbances, the distribution of local film thickness along a long side of the channel was measured for elongated bubbles flow and transition flow in the vertical rectangular microchannel with cross section 420x280 $\mu \mathrm{m}$. The distribution of a liquid film thickness along the long side of microchannel measured by LIF is shown on Figure 3 (a). The data are presented for three gas superficial velocities and fixed liquid superficial velocity $0.059 \mathrm{~m} / \mathrm{s}$. One can see that capillary forces deform the interface and maximal film thickness is observed in the middle of long side of microchannel as it was observed in [6]. Accurate film thickness measurements for whole meniscus in the channel's corner using this technique are impossible. Using high-speed video recording and method LIF, the velocities of elongated bubble were measured and compared with mixture superficial velocities.

The dependence of dimensionless film thickness on Capillary number was determined and compared with existing correlations. Figure 3 (b) shows the dependence of dimensionless liquid film thickness in the middle of a long side of the channel's cross section with length $a=420 \mu \mathrm{m}$ (scaled by microchannel hydraulic diameter) on the Capillary number. Here the experimental data of [6, 7] are presented also. The experiments were done for elongated bubble flow and transition flow. The film thickness is presented for two dimensionless distances from the bubble head $L / a$. Figure 3 (b) shows 


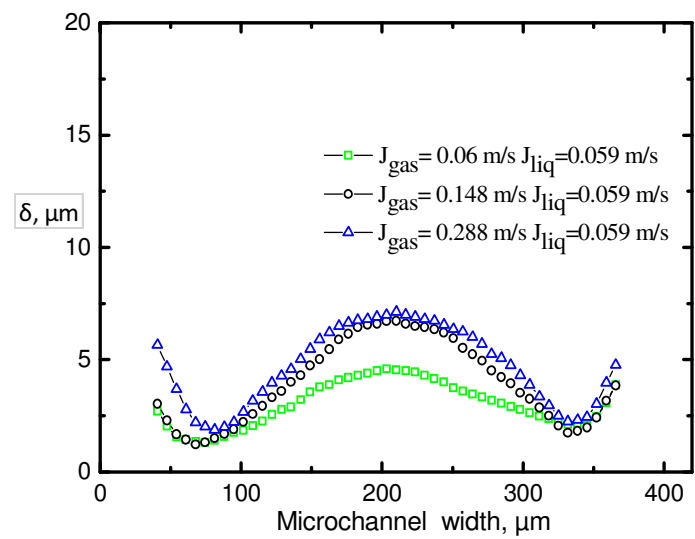

(a)

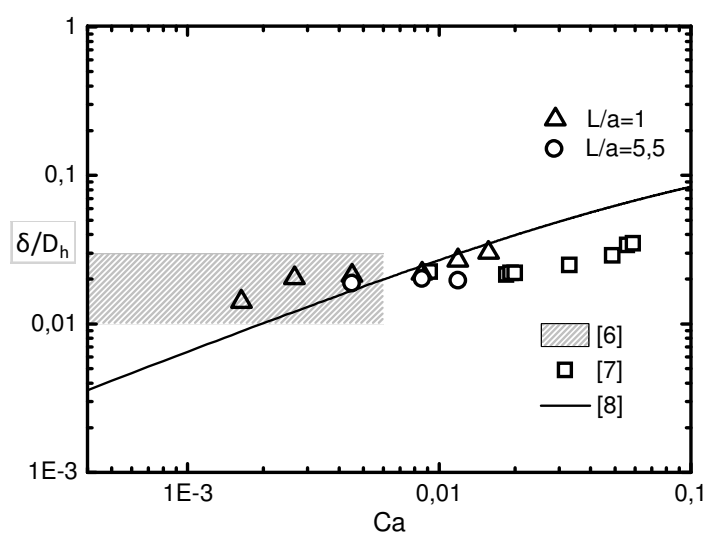

(b)

Figure 3. Distribution of liquid film thickness along long side of a microchannel measured by LIF - (a); dependence of dimensionless film thickness on the capillary number - (b).

that film thickness can be predicted by Eq. 1 only for small distance from bubble head. Increasing the distance from bubble head leads to reducing the film thickness due to capillarity induced liquid flow to the channel corners. It is the reason of the failure to comply with Taylor law in rectangular microchannel. At distance $L / a=5.5$ the experimental data show good coincidence with data [7].

\section{Conclusions}

It was observed that the elongated bubbles flow, transition flow and annular flow are the main flow patterns for rectangular microchannel with hydraulic diameter substantially less than the capillary constant. It was shown that the perspective method to define the boundary of corresponding flow patterns is statistical processing of the data obtained by laser scanning. Liquid film thickness was measured for elongated bubble flow and transition flow at two dimensionless distances from the bubble head. The LIF measurements show that the capillary forces deform the interface and maximal film thickness is observed in the middle of a long side of the microchannel's cross section. The obtained data on local film thickness confirm the failure of the Taylor law at long distance from the bubble head due to transverse capillary flows in a rectangular microchannel.

\section{Acknowledgements}

The materials of section 3 were obtained at the Kutateladze Institute of Thermophysics SB RAS by the grant from Russian Science Foundation (project 14-49-00010) and the materials of section 4 were partially supported by RFBR grant 15-08-07506-a.

\section{References}

1. M.N. Kashid, A. Renken, L. Kiwi-Minsker, Chem. Eng. Sci. 66 (2011)

2. J.W. Coleman, S. Garimella, Int. J. Heat Mass Trans. 42 (1999)

3. F.A. Kawahara, P.M.Y. Chung, M. Kawaji, Int. J. Multiphase Flow 28 (2002)

4. R. Revellin, J.R. Thome, J. Micromech. Microeng. 17 (2007)

5. I.A. Kozulin, V.V. Kuznetsov, J. Appl. Mech. Tech. Phys. 52 (2011)

6. D.M. Fries, F. Trachsel, P.R. Von Rohr, Int. J. Multiphase Flow 34 (2008)

7. W.B. Kolb, R.L. Cerro, Chem. Eng. Sci. 46 (1991)

8. P. Aussillous, D. Quere, Phys. Fluids 12 (2000) 